\title{
Reprogramming patient-derived tumor cells generates model cell lines for tuberous sclerosis-associated lymphangioleiomyomatosis
}

\section{Lisa M. Julian and William L. Stanford}

Lymphangioleiomyomatosis (LAM) is a devastating neoplasm characterised by pulmonary infiltration of abnormal smooth muscle-like cells that cause cystic replacement of the lung parenchyma, progressive tissue destruction and ultimately respiratory failure. Renal and uterine tumors as well as infiltration of LAM cell clusters within the lymphatics and venous circulation are also commonly observed, indicative of metastatic potential. LAM is defined by acquisition of inactivating mutations in one of two tumor-suppressor genes: TSC1 or TSC2 (herein referred to as $T S C 1 / 2$ ), best characterized as key negative regulators of mTORC1 signaling[1]. Germline acquisition of a TSC1/2 mutation leads to development of the multi-system disorder tuberous sclerosis complex (TSC), which includes development of low-grade skin lesions and brain tumors in both sexes and LAM (TSCLAM) in women. A sporadic form of LAM in women without TSC is caused by somatic TSC1/2 mutation. Development of LAM lesions is strongly associated with TSC1/2 loss-of-heterozygosity[1]; mouse models of renal tumorigenesis in LAM have, however, suggested pathogenic roles for TSC1/2-heterozygous cells[2,3]. Despite this genetic simplicity, appropriate cellular and animal disease models are lacking for LAM; major barriers have been the inability to propagate patientderived TSC1/2-deficient LAM cells in culture without immortalization and to generate TSC1/2-deficient animal models that recapitulate pulmonary phenotypes observed in patients. As mouse models of TSC1/2-deficiency within neural stem cell (NSC) populations have supported NSCs as a cell of origin for TSC brain tumors[1], we believe that a better understanding of the lineage that gives rise to pulmonary LAM cells will precipitate the development of effective disease models by identifying appropriate cell culture methods and lineage targeting strategies.

While the LAM cell of origin is unclear, the fact that LAM lesions are comprised of cells that express markers of the neural crest cell (NCC) lineage, including expression of smooth muscle cell (SMC) markers, suggests a NCC-SMC origin[1]. We recently established a novel cell model of LAM using a patient cell reprogramming approach focusing on the rationale that LAM cells arise from TSC1/2-deficient cells within the SMC lineage[4]. To generate this model, we reprogrammed dermal cultures obtained from either normal-appearing or angiofibroma tumor facial tissue from a TSC-LAM patient (and unaffected human controls) to induced pluripotent stem cells (iPSCs), and subsequently cultured explants of teratoma xenografts generated from these iPSCs under conditions that support SMC growth (Figure 1). The patient-derived dermal cultures possessed a germline $T S C 2$ mutation (and thus were $T S C 2^{+-}$), with second-hit mutations detected in the angiofibroma cultures at low frequency (and thus were mosaic cultures of $\mathrm{TSC}^{+-}$and $T S C 2^{-/}$cells). We established expandable cell lines from all patient samples that exhibited multiple features of pulmonary LAM cells compared to controls[4], including expression of NCC smooth muscle and melanocyte markers, elevated mTORC1 activity, deregulated autophagy and metabolic reprogramming. Notably, the angiofibroma-derived iPSCs demonstrated the most potent LAM phenotypes, suggesting epigenetic memory in tumor-derived iPSCs. These are the first non-transformed human cell line models of LAM that can be expanded in culture, and outperform the field's current gold standard human (immortalized) patient-derived cell line in our phenotypic assays. The success of this approach in generating cell lines with extensive LAM-like phenotypes provides strong evidence for the biological identity of LAM cells as aberrant cells of the SMC lineage.

An unexpected but intriguing finding of our study was that at least some TSC2 expression is required for iPSC reprogramming[4], but not maintenance of pluripotency (unpublished observations). Reflecting this, the iPSCs derived from angiofibroma cultures carried only the germline $T S C 2^{+-}$mutation, which was maintained in the teratoma-derived SMCs. This observation highlighted $T S C 2$ as a potent regulator of the cell fate decision to adopt a pluripotent state; an important question warranting further investigation is whether TSC1/2 may also function as critical regulators of fate decisions within the NCC, NCC-SMC and NSC lineages, given that the pathology driven by TSC1/2-deficiency is associated with these cell types[1, 4-6]. The potent LAM phenotypes displayed by our TSC2-heterozygous lines offer strong support for the hypothesis that $T S C 2^{+/}$cells contribute to LAM disease progression, even in the absence of TSC2 ${ }^{-}$cells[2,3]. Our model provides a critical tool to aid in unraveling 
the biology of LAM cells and to identify therapeutic avenues through drug screening initiatives. Additionally, the generation of TSC2-null iPSCs and human embryonic stem cells via CRISPR-Cas9 mediated genome engineering offers an opportunity to assess the relative contributions of TSC2-heterozygous and -homozygous cells to LAM pathology.

Our findings suggest that a TSC-LAM patient cell reprogramming approach can be used more broadly to establish TSC- and LAM-like cell lines from multiple patient biopsies. In addition to our approach of differentiating iPSCs to SMCs in teratomas, iPSCs can be differentiated in vitro into putative TSC and LAM cell types of origin (NSCs, NCCs, NCC-SMCs). An intriguing alternative approach that may maintain TSC1/2homozygous mutant cells is direct reprogramming of patient-derived somatic cells into NSCs and NCCs[7,8], forgoing a pluripotent intermediate (Figure 1). Successful reprogramming of TSC1/2-deficient cells and modeling of TSC and LAM phenotypes with these approaches would strongly support the hypotheses that the aberrant cells in TSC and LAM lesions are derived from neural and NC stem cells[1]. Importantly our study, together with recent findings that induction of TSC2-deficiency via genomic engineering in iPSC-derived NSCs can model aspects of TSC brain tumors[5,6], highlights a promising future for the use of stem cell reprogramming, hypothesis-driven differentiation, and genomic engineering approaches to develop more refined cellular models of LAM and TSC, with high likelihood for patient-specific modeling and drug discovery.

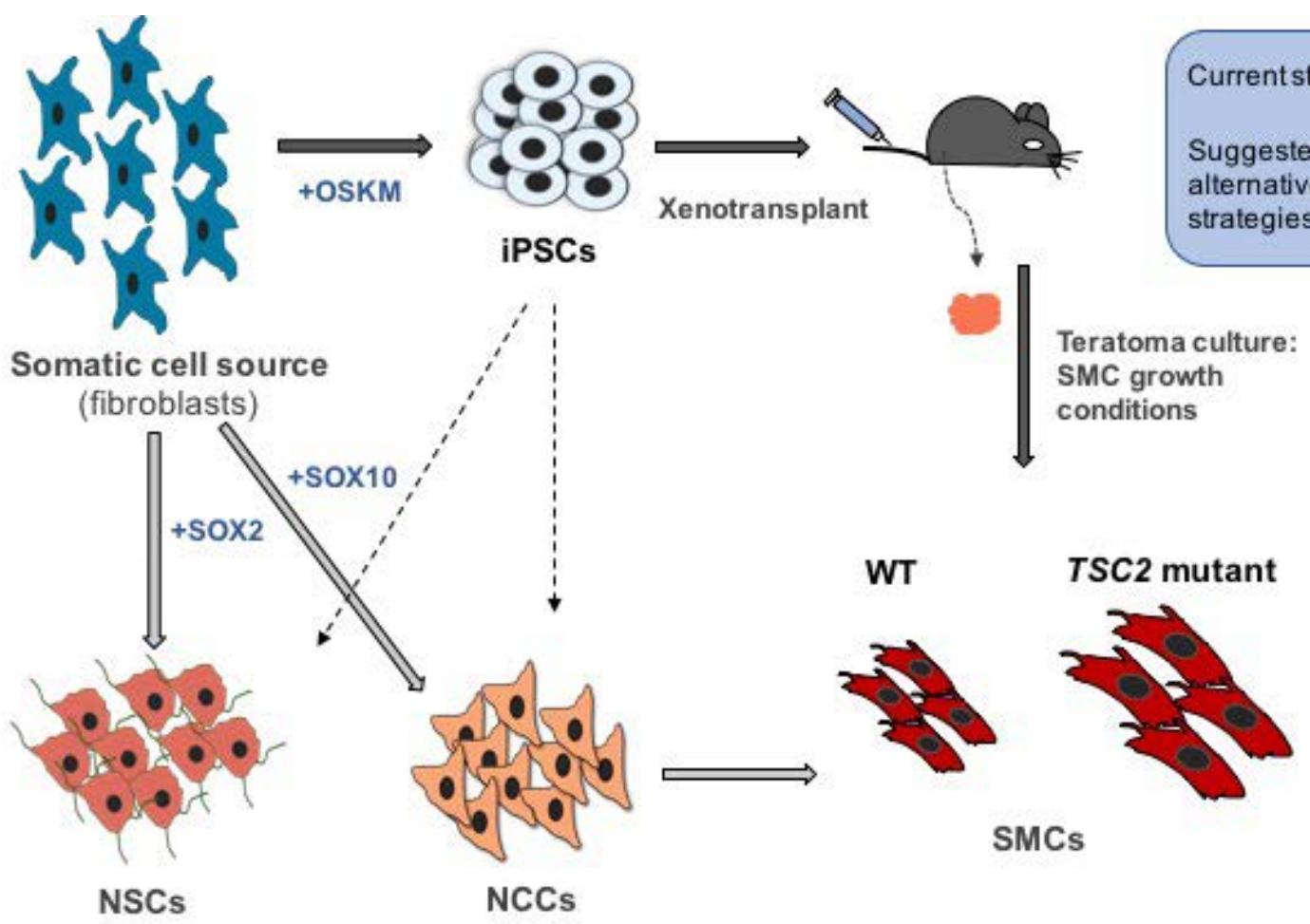

Figure 1: Cellular reprogramming strategies to establish human disease models of LAM and TSC. We recently described the establishment of a human LAM cell model[4] in which a somatic cell source (dermal fibroblasts) harvested from either a TSC-LAM patient or unaffected individuals was reprogrammed to iPSCs (via episomal OSKM factor over-expression), and explants of teratoma tumours established from these cells in immune compromised mice were cultured under smooth muscle cell (SMC) growth conditions to establish control (WT) or LAM SMC (TSC2 mutant) cell lines. TSC2 mutant SMCs exhibit LAM disease phenotypes such as increased cell size, as depicted. This strategy is amenable to establishing LAM- and TSC-like cells from additional patient-derived somatic cells or from isogenic control and TSC1/2-deficient cells established via strategies such as genomic engineering. Alternatively, TSC and LAM model cell lines could also be established by direct reprogramming of somatic cells to neural stem cells (NSCs) (i.e. by SOX2 over-expression[7]) or neural crest cells (NCCs) (i.e. by SOX10 over-expression[8]), which can be further differentiated to SMCs. NSCs and NCCs can also be differentiated directly from iPSCs. 


\section{CONFLICTS OF INTEREST}

The authors declare no conflicts of interest.

William L. Stanford: Ottawa Hospital Research Institute, Regenerative Medicine Program, Ottawa, Ontario, Canada; University of Ottawa, Ottawa, Ontario, Canada; Ottawa Institute of Systems Biology, Ottawa, Ontario, Canada

Correspondence: William L. Stanford, email wstanford@ohri. ca

Keywords: lymphangioleiomyomatosis; tuberous sclerosis complex; neural crest, patient cell reprogramming; disease modeling; disease cell of origin

Received: October 25, 2017

Published: November 02, 2017

\section{REFERENCES}

1. Delaney SP, et al. Front Cell Dev Biol. Frontiers. 2014; 2:69.

2. D'Armiento J, et al. Cancer Res. 2016; 76:844-54.

3. Wilson C, et al. Cancer Res. 2006; 66:7934-8.

4. Julian LM, et al. Cancer Res. 2017; 77:5491-502.

5. Costa V, et al. Cell Rep. 2016; 15:86-95.

6. Ebrahimi-Fakhari D, et al. Cell Rep. 2016; 17:1053-70.

7. Ring KL, et al. Cell Stem Cell. 2012; 11:100-9.

8. Kim YJ, et al. Cell Stem Cell. 2014; 15:497-506.

Copyright: Julian et al. This is an open-access article distributed under the terms of the Creative Commons Attribution License 3.0 (CC BY 3.0), which permits unrestricted use, distribution, and reproduction in any medium, provided the original author and source are credited. 